OPEN ACCESS

Edited by:

Harry T. Whelan,

Medical College of Wisconsin, USA

Reviewed by:

Gouri Rao Passi,

Choithram Hospital and Research Centre, India

Kumar Sannagowdara,

Medical College of Wisconsin, USA

*Correspondence:

Anna Maria Lavezzi anna.lavezzi@unimi.it

Specialty section: This article was submitted to Neuropediatrics, a section of the journal Frontiers in Neurology

Received: 28 July 2015 Accepted: 05 October 2015 Published: 19 October 2015

Citation: Lavezzi AM (2015) A new theory to explain the underlying pathogenetic mechanism of sudden infant death syndrome.

Front. Neurol. 6:220 doi: 10.3389/fneur.2015.00220

\section{A new theory to explain the underlying pathogenetic mechanism of sudden infant death syndrome}

\author{
Anna Maria Lavezzi* \\ "Lino Rossi" Research Center for the Study and Prevention of Unexpected Perinatal Death and SIDS, Department of \\ Biomedical, Surgical and Dental Sciences, University of Milan, Milan, Italy
}

The author, on the basis of numerous studies on the neuropathology of SIDS, performed on a very wide set of cases, first highlights the neuronal centers of the human brainstem involved in breathing control in perinatal life, with the pontine Kölliker-Fuse nucleus (KFN) as main coordinator. What emerges from this analysis is that the prenatal respiratory movements differ from those post-natally in two respects: (1) they are episodic, only aimed at the lung development and (2) they are abolished by hypoxia, not being of vital importance in utero, mainly to limit the consumption of oxygen. Then, as this fetal inhibitory reflex represents an important defense expedient, the author proposes a new original interpretation of the pathogenetic mechanism leading to SIDS. Infants, in a critical moment of the autonomic control development, in hypoxic conditions could awaken the reflex left over from fetal life and arrest breathing, as he did in similar situations in prenatal life, rather than promote the hyperventilation usually occurring to restore the normal concentration of oxygen. This behaviour obviously leads to a fatal outcome. This hypothesis is supported by immunohistochemical results showing in high percentage of SIDS victims, and not in age-matched infant controls, neurochemical alterations of the Kölliker-Fuse neurons, potentially indicative of their inactivation. The new explanation of SIDS blames a sort of auto-inhibition of the KFN functionality, wrongly arisen with the same protective purpose to preserve the life in utero, as trigger of the sudden infant death.

Keywords: SIDS, pathogenesis, brainstem, respiratory network, fetal breathing, Kölliker-Fuse nucleus, BDNF, NeuN

\section{INTRODUCTION}

\section{Most Reliable Definition and Hypothesis on SIDS Pathogenesis}

The best known definition of SIDS is "the sudden unexpected death of an infant $<1$ year of age, with onset of the fatal episode apparently occurring during sleep, that remains unexplained after a thorough investigation, including performance of a complete autopsy and review of the circumstances of death and the clinical history" (1). The causes are still unknown, although several even conflicting hypotheses of the underlying mechanisms of SIDS have been proposed (2). The most reliable seems to be the "triple risk hypothesis," which predicts that fetal brain development of infants who subsequently succumb to SIDS is abnormal, leaving them unable to respond appropriately to stressors during a vulnerable period of the autonomic control (3). Consistent with this assumption, many studies have reported a high incidence of morphological abnormalities and biochemical defects of neurotransmission, particularly serotonergic, in the brainstem of SIDS victims compared 


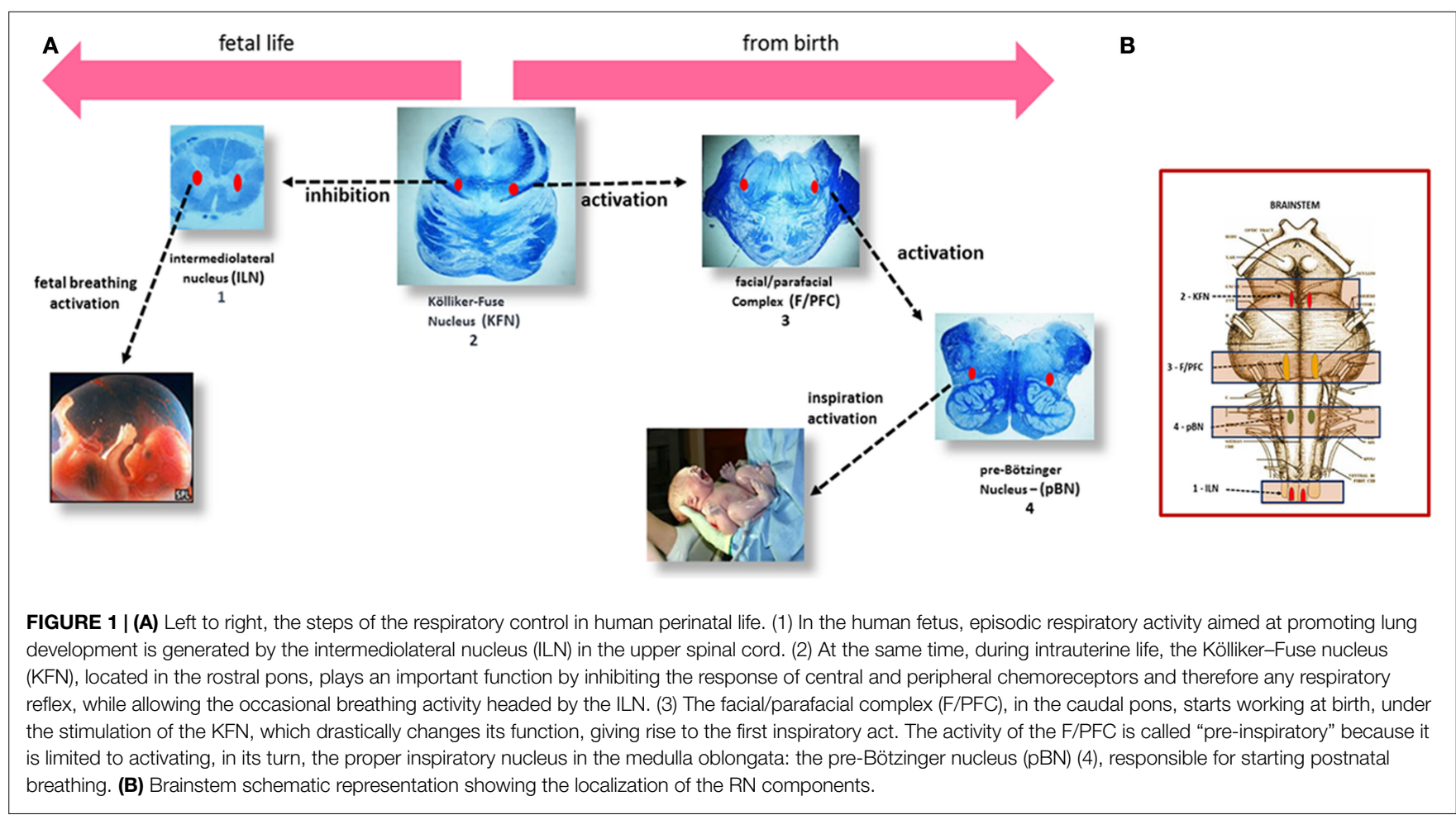

with control infants dying of other causes $(4,5)$. This brain region includes the main nuclei and structures that coordinate the vital activities, such as cardiovascular function and breathing, before and after birth.

\section{A Rightful Observation}

But if the placenta is the effective source of oxygen in fetal life, what is the significance of the respiratory activity in prenatal life? The answer seems to be that the fetus must train so as to be ready to put the lungs to use, once outside the womb. At the time of birth, he will take a few moments to dilate the lungs and begin to breathe. If he fails, survival is threatened. So, in utero intermittent breathing movements occur with the main purpose of adequately developing the respiratory system, essential as soon as postnatal life begins (6). The full functionality of the lungs and respiratory muscles acquired will allow the autonomous ventilatory activity that the newborn needs to survive to start up (7).

\section{Breathing Behavior Before and After Birth in Hypoxic Conditions}

A very interesting phenomenon was observed by low-voltage cortical electrical tests in experimental studies on sheep fetuses (8, 9), and also by ultrasound real-time scanning in human fetuses (10): if the amount of oxygen through the placenta decreases (for whatever reason), the fetus immediately suspends pulmonary movements. Precisely, when the partial pressure of oxygen falls below $16-18 \mathrm{mmHg}$, respiratory activity in utero stops, not being a vital function, but only to limit the consumption of oxygen. This is a defense mechanism of the fetus, a way to save energy, because oxygen must go first and foremost to the brain and heart to ensure life.
On the contrary, hypoxia induces hyperventilation in newborns, mainly in the arousal phase from sleep, through a rise in the amplitude and frequency of pulmonary movements, to restore the normal concentration of plasmatic gas and above all of oxygen (11).

\section{A New Plausible Hypothesis on SIDS Pathogenesis}

And it is at this point, on the basis of the above considerations, that I propose a new perspective that could provide a physiological explanation of SIDS. Infants, in a critical moment of the autonomic control development, could awaken a conditioned reflex left over from fetal life: after birth, a situation of lack of oxygen (due to a prone sleep position, nicotine absorption, or any other reason) could induce a vulnerable baby to arrest breathing, he did in similar situations in prenatal life, and, therefore, die. Very probably, being used to being oxygenated by the placenta for 9 months, this ancient instinctive survival behavior could remain registered in the brain, but becoming fatal after birth.

How could the brainstem centers checking the respiratory function be involved in this intrinsic devastating reflex leading to SIDS?

\section{The Brainstem Respiratory Network}

Previous studies, performed at the "Lino Rossi" Research Center of the Milan University, have identified specific nuclei and structures designated to control the breathing, hitherto highlighted only in experimental animals. Given, obviously, the impossibility of performing experiments in humans, the homologous nuclei were identified on the basis of morphological criteria of similarity with regard to the location, the cytoarchitecture, and 


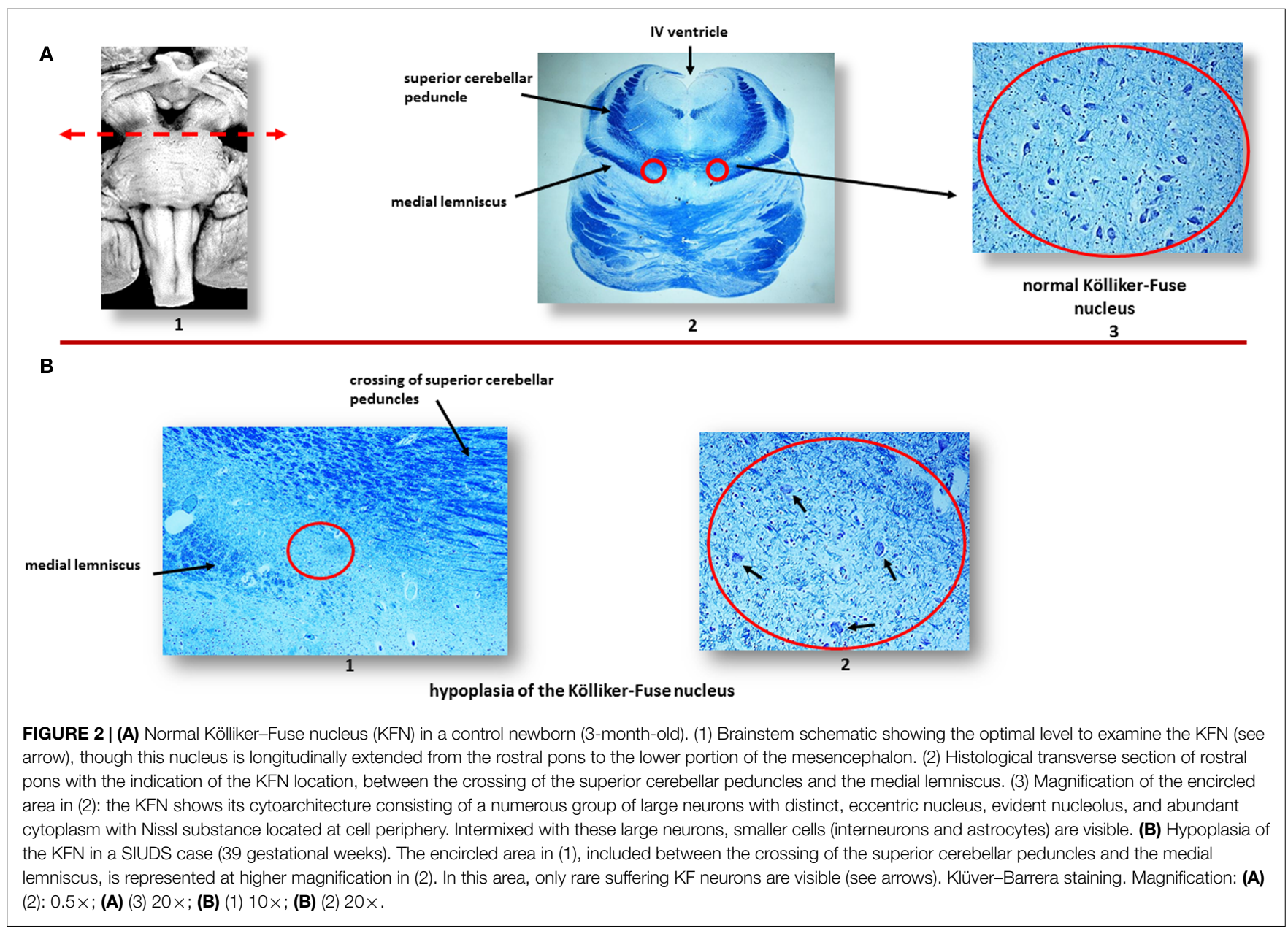

number of neurons and applying, when possible, immunohistochemical methods to highlight the same neurotransmitters and receptors recognized as specific for several structures, above all in rats. Through this original methodology, the Kölliker-Fuse nucleus (KFN), the facial/parafacial complex (F/PFC), the preBötzinger ( $\mathrm{pBN}$ ) nucleus in the pons/medulla oblongata, and the intermediolateral nucleus (ILN) in the spinal cord were defined (12-15). These nervous centers are linked together via interneuronal synapses in a "respiratory network" (RN), and can modulate one another. I propose a scheme (Figure 1) to illustrate the human breathing control mechanism in perinatal life, indicating the more representative brainstem histological sections where the $\mathrm{RN}$ structures are well analyzable, depicted in a chronologically functional sequence, as explained in the legend.

\section{Role of the Kölliker-Fuse Nucleus in the RN}

Essentially, the pulmonary activity is largely dependent on sensory inputs from the ILN in prenatal life and from the F/PFC from birth, being both modulated by the KFN, which therefore represents the breathing filmmaker. Its activity, changing from fetal to postnatal life thanks to a skillful interplay of activation and inactivation of its GABAergic inhibitory and glutamatergic excitatory neurons (16), is fundamental. In hypoxic conditions, a normal fully functional KFN abolishes the rhythmic activity of the
ILN in fetuses and greatly accelerates the ventilatory function of the F/PFc in newborns, with the same aim of safeguarding life.

This chief central function exerted by the KFN is supported by experimental studies in fetal lambs performed by brainstem transactions at various levels (17). Results demonstrated the existence of a locus in the rostral lateral pons whose integrity is essential for the depression of breathing during hypoxia in utero. The authors suggested that this structure, even if not well-identified anatomically in the sheep brainstem, could correspond to the KFN, previously defined as the core of the "pneuomotaxic center" (18).

\section{Histological and Immunohistochemical Personal Findings in SIDS}

The new etiopathogenic interpretation of SIDS here presented is validated by the findings obtained over many years of personal studies on sudden intrauterine unexplained death syndrome (SIUDS) and SIDS. The "Lino Rossi" Research Center of the Milan University has, in fact, collected a large number of sudden fetal and infant death cases, in application of the Italian Law $31 / 2006$ "Regulations for diagnostic post mortem investigation in victims of the SIDS and unexpected fetal death" (19). This law stipulates that all infants who died suddenly in Italian regions within the first year of life and fetuses that die after the 25th week of gestation without any apparent cause, must be rapidly 
A

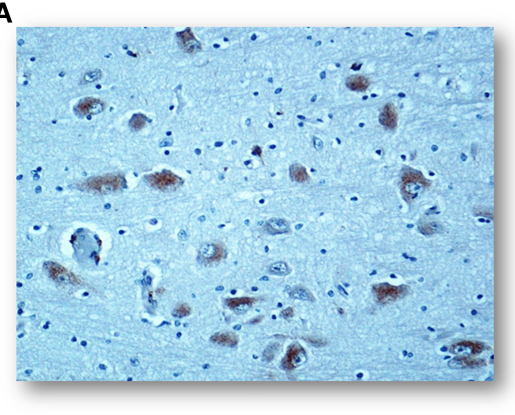

B

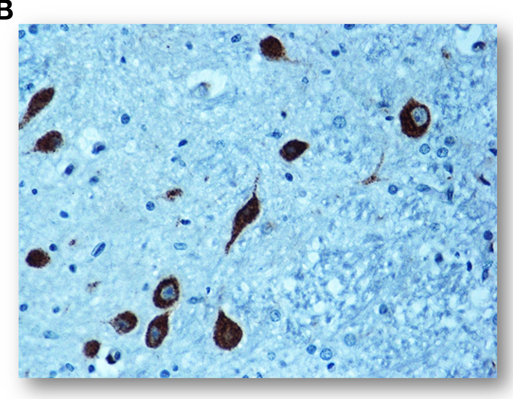

FIGURE 3 | (A) Regular negative/weakly immunopositive BDNF expression in the cytoplasm of the KF neurons in a control infant (3-month-old). (B) Intense immunopositivity of the KF neurons with dark brown cytoplasmatic staining in a SIDS case, died at 4 months of life. BDNF immunostaining. Magnification: $20 \times$.

A

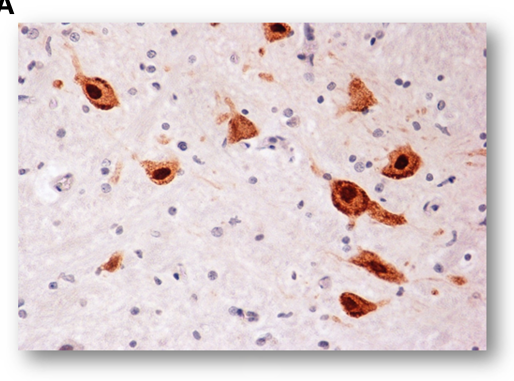

B

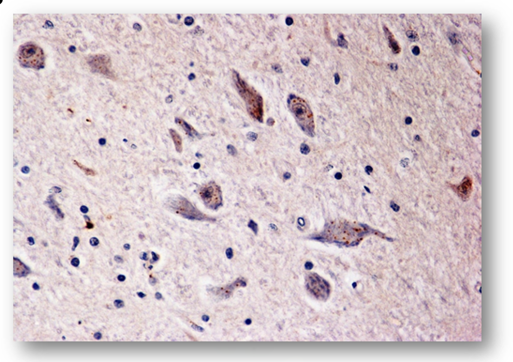

FIGURE 4 | (A) NeuN-immunoreactive neurons of the KFN in an infant of the control group (2-month-old). The staining is particularly strong in the neuronal nucleus but also the cytoplasm and the proximal part of the processes are immunoreactive, though to a lesser intensity. (B) NeuN-immunonegative KF neurons in an age-matched infant died of SIDS. NeuN immunostaining. Magnification: $20 \times$.

submitted, after obtaining informed parental consent, to in-depth anatomopathological examination, particularly of the autonomic nervous system, with the components of the $\mathrm{RN}$ as the main object of study.

Permission from the Ethics Committee was not required for this study as the "Lino Rossi" Research Center is the national referral center for the sudden unexplained fetal and infant deaths, in accordance with the above mentioned Law 31/2006.

In a large number of SIUDS (up to now no. 95 cases, aged from 25 to 40 gestational weeks), SIDS (no. 150 cases, 1- to 11-month-old), and age-matched controls (no. 35 fetuses and 30 infants), I found developmental hypoplasia of the KFN only in late fetal unexplained deaths (20\%), never in newborns and infants (Figure 2) (20). This means that a normal structure of this nucleus is absolutely essential for vital breathing from birth. However, in a high percentage of SIDS cases (no.105, 70\%), and not in infant of the control group, despite a normal morphological cytoarchitecture of the KFN, neurochemical alterations, such as an unusual immunopositivity of the brain-derived neurotrophic factor (BDNF) and a decreased expression of the neuronal nuclear antigen (NeuN) were highlighted in the KF neurons (Figures 3 and 4$)(21,22)$.

The BDNF is a member of the neurotrophin class of growth factors required for the normal development and maturation of specific brainstem centers involved in respiratory control, and therefore expressed in both inhibitory and excitatory neurons of the KFN during the biphasic breathing modulation in intrauterine life $(23,24)$. After birth, the BDNF is unexpressed in the KFN in human control newborns, while the positivity, as observed in SIDS cases, seems to hinder any ventilatory activity.

By contrast, the NeuN is a protein expressed in post-mitotic functional neurons (25). Then, the specific immunohistochemical method can be applied in neuropathological studies to highlight the physiological status of the neurons (26). While intense NeuN expression is shown by healthy neurons, a considerably reduced NeuN immunopositivity in postnatal life can be indicative of a degeneration of differentiated neurons.

\section{Correlation of Findings with Nicotine Absorption}

In general, morphological and functional brainstem abnormalities resulted significantly related to severe injuries, such as hypoxia. Indeed, the majority of mothers, and often also fathers, of infants with altered KFN development were found to be smokers, either by their own admission, or based on positivity of the cotinine test in the hair of the victims. In case of maternal smoking in pregnancy, carbon monoxide, a gaseous combustion product of nicotine, easily crosses the placental barrier by passive diffusion, and binds to fetal hemoglobin, so giving rise to carboxyhemoglobin $(\mathrm{COHb})$. The same chemical bond occurs when an 
infant inhales considerable amounts of environmental smoke. $\mathrm{COHb}$ is not able to release oxygen into tissues, leading to a general hypoxic status (27). Besides, nicotine is one of the few lipidsoluble substances that can go beyond the blood-brain barrier and induce specific molecular alterations in the DNA, RNA, and antigenic proteins of the nervous cells $(28,29)$.

\section{CONCLUSION}

Based on these observations, I propose that during the first months of life, when a predisposed subject is particularly vulnerable, hypoxia can unexpectedly switch on again the ancestral fetal behavior designed to suspend respiration, being this as a nonessential activity, through a functional degeneration of the KF

\section{REFERENCES}

1. Krous HF, Beckwith JB, Byard RW, Rognum TO, Bajanowski T, Corey T, et al. Sudden infant death syndrome and unclassified sudden infant deaths: a definitional and diagnostic approach. Pediatrics (2004) 114:234-8. doi:10.1542/ peds.114.1.234

2. Goldwater PN. A perspective on SIDS pathogenesis. The hypotheses: plausibility and evidence. BMC Med (2011) 9:64. doi:10.1186/1741-7015-9-64

3. Filiano JJ, Kinney HC. A perspective on neuropathologic findings in victims of the sudden infant death syndrome: the triple-risk model. Biol Neonate (1994) 65:194-7. doi: $10.1159 / 000244052$

4. Paine SM, Jacques TS, Sebire NJ. Review: neuropathological features of unexplained sudden unexpected death in infancy: current evidence and controversies. Neuropathol Appl Neurobiol (2014) 40:364-84. doi:10.1111/nan.12095

5. Kinney HC. Neuropathology provides new insight in the pathogenesis of the sudden infant death syndrome. Acta Neuropathol (2009) 117:247-55. doi:10. 1007/s00401-009-0490-7

6. Ten Have-Opbroek AA. The development of the lung in mammals: an analysis of concepts and findings. Am J Anat (1981) 162:201-19. doi:10.1002/aja. 1001620303

7. Wigglesworth JS, Dessai R. Is fetal respiratory function a major determinant of perinatal survival? Lancet (1982) 1:264. doi:10.1016/S01406736(82)90986-2

8. Boddy K, Dawes GS, Fisher R, Pinter S, Robinson JS. Foetal respiratory movements, electrocortical and cardiovascular responses to hypoxaemia and hypercapnia in sheep. J Physiol (1974) 243:599-618. doi:10.1113/jphysiol.1974. sp010768

9. Dawes GS. Fetal breathing movements in normoxia and in hypoxia. In: Künzel W, et al., editors. Oxygen: Basis of the Regulation of Vital Functions in the Fetus. (Vol. 9), Berlin, Heidelberg: Springer-Verlag (1992). p. 46-51.

10. Patrick J, Challis J. Measurement of human fetal breathing movements in healthy pregnancies using a real-time scanner. Semin Perinatol (1980) 4:275-86.

11. Thach BT, Lijowska A. Arousals in infants. Sleep (1996) 19(Suppl):S271-3.

12. Lavezzi AM, Ottaviani G, Rossi L, Matturri L. Cytoarchitectural organization of the parabrachial/Kölliker-Fuse complex in man. Brain Dev (2004) 26:316-20. doi:10.1016/j.braindev.2003.09.002

13. Lavezzi AM, Matturri L. Hypoplasia of the parafacial/facial complex: a very frequent finding in sudden unexplained fetal death. Open Neurosci $J$ (2008) 2:1-5. doi:10.2174/1874082000802010001

14. Lavezzi AM, Matturri L. Functional neuroanatomy of the human pre-Bötzinger complex with particular reference to sudden unexplained perinatal and infant death. Neuropathology (2008) 28:10-6. doi:10.1111/j.1440-1789.2007. 00824.x

15. Lavezzi AM, Corna M, Mehboob R, Matturri L. Neuropathology of the intermediolateral nucleus of the spinal cord in sudden unexplained perinatal and infant death of raphe nuclei and serotonin transporter gene promoter polymorphism. Ped Res (2009) 66:22-7. doi:10.1016/j.ijdevneu.2010.01.001

16. Damasceno RS, Takakura AC, Moreira TS. Regulation of the chemosensory control of breathing by Kölliker-Fuse neurons. Am J Physiol Regul Integr Comp Physiol (2014) 307:R57-67. doi:10.1152/ajpregu.00024.2014 neurons and then a depression of the central respiratory control. This is a protective reflex in the womb but a rapidly fatal device in postnatal life.

Clearly, further studies are required, specifically designed to address this exciting theory that offers consistent assumptions to explain the pathogenetic mechanism occurring in a substantial group of SIDS.

\section{ACKNOWLEDGMENTS}

This study was supported by the Italian Health's Ministry in accordance with the Law 31/2006 "Regulations for Diagnostic Post Mortem Investigation in Victims of Sudden Infant Death Syndrome (SIDS) and Unexpected Fetal Death.”

17. Gluckman PD, Johnston BM. Lesions in the upper lateral pons abolish the hypoxic depression of breathing in unanaesthetized fetal lambs in utero. $J$ Physiol (1987) 382:373-83. doi:10.1113/jphysiol.1987.sp016372

18. Cohen MI. Neurogenesis of respiratory rhythm in the mammal. Physiol Rev (1979) 59:1105-73

19. Constitution of the Italian Republic Law No 31. Regulations for diagnostic postmortem investigation in victims of sudden infant death syndrome (SIDS) and unexpected fetal death. Official Gazette Italian Republic, General Series (2006) 34:4.

20. Matturri L, Lavezzi AM. Unexplained stillbirth versus SIDS: common congenital diseases of the autonomic nervous system - pathology and nosology. Early Hum Dev (2011) 87:209-15. doi:10.1016/j.earlhumdev.2010.12.009

21. Lavezzi AM, Corna MF, Matturri L. Disruption of the brain-derived neurotrophic factor (BDNF) immunoreactivity in the human Kölliker-Fuse nucleus in victims of unexplained fetal and infant death. Front Hum Neurosci (2014) 8:648. doi:10.3389/fnhum.2014.00648

22. Lavezzi AM, Corna MF, Matturri L. Neuronal nuclear antigen (NeuN): a useful marker of neuronal immaturity in sudden unexplained perinatal death. J Neurol Sci (2013) 329:45-50. doi:10.1016/j.jns.2013.03.012

23. Balkowiec A, Katz DM. Brain-derived neurotrophic factor is required for normal development of the central respiratory rhythm in mice. J Physiol (1998) 510:527-33. doi:10.1111/j.1469-7793.1998.527bk.x

24. Kron M, Mörschel M, Reuter J, Zhang W, Dutschmann M. Developmental changes in brain-derived neurotrophic factor-mediated modulations of synaptic activities in the pontine Kölliker-Fuse nucleus of the rat. J Physiol (2007) 583:315-27. doi:10.1113/jphysiol.2007.134726

25. Sarnat HB, Nochlin D, Born DE. Neuronal nuclear antigen (NeuN): a marker of neuronal maturation in early human fetal nervous system. Brain Dev (1998) 20:88-94. doi:10.1016/S0387-7604(97)00111-3

26. Wolf HK, Buslei R, Schmidt-Kastner R, Schmidt-Kastner PK, Pietsch T, Wiestler OD, et al. NeuN: a useful neuronal marker for diagnostic histopathology. $J$ Histochem Cytochem (1996) 44:1167-71. doi:10.1177/44.10.8813082

27. Prockop LD, Chichkova RI. Carbon monoxide intoxication: an updated review. J Neurol Sci (2007) 262:122-30. doi:10.1016/j.jns.2007.06.037

28. Lavezzi AM, Ottaviani G, Matturri L. Adverse effects of prenatal tobacco smoke exposure on biological parameters of the developing brainstem. Neurobiol Dis (2005) 20:601-7. doi:10.1016/j.nbd.2005.04.015

29. Lichtensteiger W, Ribary U, Schiumpf M, Odermatt B, Widemer HR. Prenatal adverse effects of nicotine on the developing brain. Prog Brain Res (1988) 73:137-57. doi:10.1016/S0079-6123(08)60502-6

Conflict of Interest Statement: The author declares that the research was conducted in the absence of any commercial or financial relationships that could be construed as a potential conflict of interest.

Copyright (C) 2015 Lavezzi. This is an open-access article distributed under the terms of the Creative Commons Attribution License (CC BY). The use, distribution or reproduction in other forums is permitted, provided the original author(s) or licensor are credited and that the original publication in this journal is cited, in accordance with accepted academic practice. No use, distribution or reproduction is permitted which does not comply with these terms. 